\title{
Development of Algorithm for Identification of Area for Maximum Coverage and Interference
}

\author{
Janak Gupta
}

\author{
Pankaj Kumar
}

\begin{abstract}
In this paper we consider the following problem: Given a set $n$ shops of Store1 in the plane P than how many minimum shops of Store2 to be open for the maximum coverage and interference Store1 Shops. The problem is solve using the Stabbing disk induced by points on the plane P. For a point set $\mathrm{P}$, where no two points have the same $\mathrm{x}$ or $\mathrm{y}$ coordinates, derive an upper bound on the size of the stabbing set of $\left(\begin{array}{l}n \\ 2\end{array}\right)$ axis-parallel rectangles induced by each pair of points $a, b \in P$ as the diagonal of the rectangles. For a point set $\mathrm{P}$ in convex position, derive a lower bound on the size of the stabbing set $\left(\begin{array}{l}\mathrm{n} \\ 2\end{array}\right)$ axis-parallel rectangles induced by each pair of points $\mathrm{a}, \mathrm{b} \in \mathrm{P}$ as the diagonal of the rectangles.
\end{abstract}

\section{Keywords}

Stabbing, Proximity Graph, Gabriel Graphs, Triangulation, Neighborliness, Convex hull,rectangle stabbing.

\section{INTRODUCTION}

Area identification for maximum coverage and interference is important parts in different market field. If any brand store 1 have there old shops in some region and there market is already established than if another brand store 2 want to open there shops in that area so that it cover maximum area and maximum interfere the market of brand store 1 .

This problem come in different area as like in army they have to stab (mark) that point from where they cover the maximum area for the bunkers. So the stabbing is done by circles and rectangles induced by point sets. As the stabbing is the marking the points that is to be selected. The brand store owner also wants to open minimum shops in that area so that it can cover maximum area. . As the brand store is marked as point and for stabbing these points we can use disks and rectangles as for the marking. There are different graphs that are to be used like proximity graph, Gabriel graph, Delaunay graph, witness Gabriel graph and witness Delaunay graphs etc. Identify the area that can cover maximum area is to be marked when the disks are to be induced by point sets. These point sets is joined by the line segment and treated these line segment as diameter we can solve this problem. There is another way by treated these line segment as chord than some result comes. As far as rectangles concerned they treated line as diagonal and make the axis-parallel rectangle. We can use convex hull position as for the boundary value. The convex hull is the boundary points in which all the points are to be covered and the other point's lies inside this convex hull boundary. These all terms are to be used in this for solving the problem. Planarity of graph is to be taken that means all the points are in same plane.

The aim is to find optimal solution of above problems using the stabbing disk induced by points on the plane. Let the number of Store1 are ' $n$ ' with point set P. Initially to get the solution of the problem the disk is defined by the pairs of points as diameter of circle and diagonal of rectangle.

For the neighbourliness concept proximity are used Proximity graph can be intuitively defined as follows: given a point $\mathrm{P}$ in the plane, the vertices of the graph $\mathrm{s}$, there is an edge between a pair of vertices $\mathrm{p}, \mathrm{q} \in \mathrm{P}$ if they satisfy some particular notion of neighbourliness.

Proximity graph [1] is a graph where the edges between the vertices of the graphs depend on the neighbourlines of the vertices. Proximity graph can be intuitively defined as follows: given a point $\mathrm{P}$ in the plane, the vertices of the graph $s$, there is an edge between a pair of vertices $p, q \in P$ if they satisfy some particular notion of neighborliness.

In the case of Delaunay Graphs. DG(P) [6], the region of influence of a pair of vertices $a, b$ is the set of closed disks $D_{a b}$ with chord $\overline{a b}$.

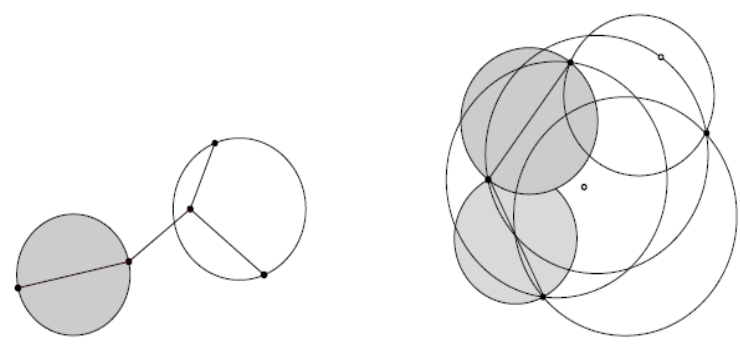

Figure 1 Gabriel graph.

The vertices defining a shaded disk are adjacent and the pairs defining the shaded disks are adjacent because their disk does not contain any other vertex, in contrast to the other vertices defining the shaded disk .Right: Witness Gabriel graph .Black points are the vertices of the graph, white points are the witnesses.Each pair of vertices defining a shaded disk are adjacent and the pairs defining the unshaded disks are

\section{AREA IDENTIFICATION BY STABBING RECTANGLES}

\subsection{Stabbing Rectangles Induced By Points On The Plane}

For a given point set $\mathrm{P}$ of $\mathrm{n}$ points with no two $\mathrm{x}$ or $\mathrm{y}$ coordinates same, for any pair of points $p, q \in P$, there is an axis parallel rectangle in $S$ with one of its diagonal as $\overline{p q}$. So the set of object $\mathrm{S}$ for point set $\mathrm{P}$ contains $\left(\begin{array}{l}n \\ 2\end{array}\right)$ axis-parallel rectangles, each corresponding to each pair of points. 


\subsection{Axis-parallel Rectangles Induced by Pair of Points as diameter}

In this section, we will argue a 4-approximation algorithm for the size of stabbing set of axis-parallel rectangles for a set of points $\mathrm{P}$ in convex position.

We start with the upper bound for the size of the stabbing set.

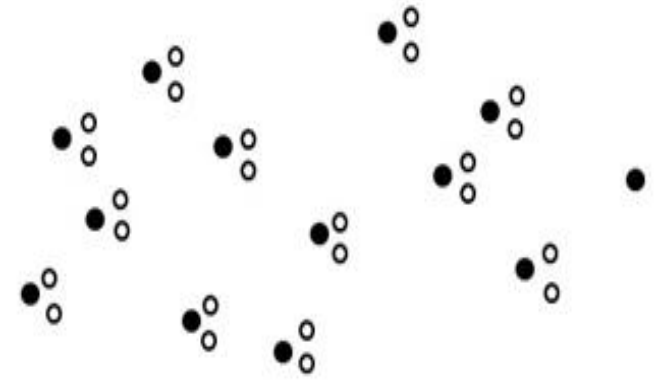

Figure 2 making sure that all rectangles have been stabbed

Theorem 2.1. $2 n-2$ stabbing pints are always sufficient to stab all the rectangles of $\mathrm{S}$ for a point set $\mathrm{P}$ having $\mathrm{n}$ points in general position.

Proof: We assume that no two points have same $\mathrm{x}$ or $\mathrm{y}$ coordinate. We place two stabbing points one slightly above and another one slightly below to the right of each point of $P$ except the point having maximum $\mathrm{x}$ coordinate (refer Figure 2 ). If we consider $p$ as origin, the stabbing point above $p$ will stab all the rectangles corresponding to diagonal $\overline{p q}$ where $q$ belongs to the first quadrant and the stabbing point below $\mathrm{p}$ will stab all the rectangles corresponding to diagonal $\overline{\mathrm{pq}}$ where $\mathrm{q}$ belongs to the fourth quadrant. Every rectangle with diagonal determined by two points of $\mathrm{P}$ will contain a stabbing point. So we have proved that $2 \mathrm{n}-2$ stabbing point will be sufficient to stab all the rectangles.

Now we will give a lower bound for the stabbing set of $\mathrm{s}$ when the point of $\mathrm{P}$ are in convex position. We construct an intersection graph $\mathrm{G}(\mathrm{V}, \mathrm{E})$ for the rectangles corresponding to the edge of the convex hull of the point of $\mathrm{P}$. we call all such rectangles as boundary rectangles. The set $\mathrm{V}$ contains one vertex for each boundary rectangle. There is an edge between two vertices of $G$ if and only if the rectangles corresponding to vertices intersect.
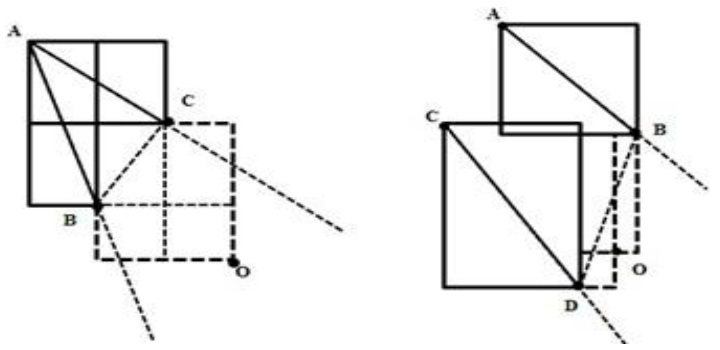

Figure 3 Left: No three rectangles corresponding to adjacent edges intersect. Right: No three rectangles corresponding to non-adjacent edges intersect.

Theorem 2.2. Let $\mathrm{P}$ be a point set of $\mathrm{n}$ points and the points are convex position. The intersection graph $G$ of the rectangles corresponding to the edges of the convex hull (boundary rectangles) of $\mathrm{P}$ does not contain any clique of size three or more.

Proof: to prove that there is no clique in G of size three or more, we will prove that no three rectangles intersect together.
First we need to find out when the two boundary rectangles intersect. There will be two cases either the rectangle will be corresponding to adjacent edges or they will be corresponding to non-adjacent edges. In the first case when the rectangles are corresponding to adjacent edges, let the rectangles corresponding to edges $\mathrm{AB}$ (say R1) and $\mathrm{AC}$ (say R2) intersect. In this case the point $\mathrm{B}$ and $\mathrm{C}$ both will lie in the same quadrant if we consider A as origin. Due to the convexity condition all other points must lie in the region $\mathrm{R}$ bounded by lines passing through $\mathrm{AB}, \mathrm{AC}$ and $\mathrm{BC}$ (say Figure 3). Let point $\mathrm{O}$ lies in the region $\mathrm{R}$. then we can claim that the rectangle corresponding to edge $\mathrm{BO}$ cannot intersect with the rectangle $\mathrm{R} 1$, as point $\mathrm{A}$ and $\mathrm{O}$ lies in the different quadrant if we consider point B as origin. Similarly, we can prove that the rectangle corresponding to edge $\mathrm{AC}$ and $\mathrm{CO}$ cannot intersect. The rectangle corresponding to an edge between two points from $\mathrm{R}$ cannot intersect with rectangles $\mathrm{R} 1$ and $\mathrm{R} 2$, as both the point will lie to the right to $\mathrm{B}$ and below to $\mathrm{C}$. So we have seen that in the case when two rectangles corresponding to adjacent edge intersect there does not exist third rectangle which can intersect with both of them. Now we consider the second case when R1 and R2 are corresponding to nonadjacent edges $\mathrm{AB}$ and $\mathrm{CD}$ (refer Figure 3 ). There will be two regions where the others point can be, the region bounded by lines $\mathrm{AB}, \mathrm{CD}$ and $\mathrm{Ac}$ and the region bounded by the lines $\mathrm{AB}$, $\mathrm{CD}$ and $\mathrm{BD}$. Let us consider the region bounded by $\mathrm{AB}, \mathrm{CD}$ and $\mathrm{BD}$ (say $\mathrm{R}$ ). Let $\mathrm{O}$ be a point in $\mathrm{R}$. We can claim that rectangle corresponding to edge $\mathrm{OB}$ cannot intersect with $\mathrm{R}$, as $\mathrm{A}$ and $\mathrm{O}$ will lie in the different quadrant if we consider $\mathrm{B}$ as origin. Similarly, it can be proved that the rectangle corresponding to edge OD cannot intersect with $\mathrm{R} 2$. The rectangle corresponding to two points from $\mathrm{R}$ will not intersect with R1 and R2, as both the points will lie to the right of $\mathrm{D}$ and below $\mathrm{B}$. So we have proved that there is no point or pair of points in $\mathrm{R}$ which can make three rectangles intersect. Similarly, in can be proved for other region too. So as claimed we have proved that there will be no three boundary rectangles intersecting in a common region. This, in turn, implies that there will be no clique of size three or more in graph $\mathrm{G}$.

Theorem 3.3. For a point set $\mathrm{P}$ in convex position. We derive a 4-factor approximation algorithm for the minimum size of the stabbing set of $\left(\begin{array}{l}n \\ 2\end{array}\right)$ Axis-parallel rectangles induced by each pair of points $a, b \in P$ as the diagonal of the rectangles.

Proof . By Theorem 2.1. We can say that there will be atleast $\mathrm{n} / 2$ disjoint rectangles in $\mathrm{S}$. This gives a lower bound on the optimal solution. The above lower bound coupled with the constructive proof of upper bound of $2 n-2$ in the Theorem 2.2gives us a 4 -factor approximation algorithm.

Now we will improve the lower bound of stabbing set, for the points in general position.

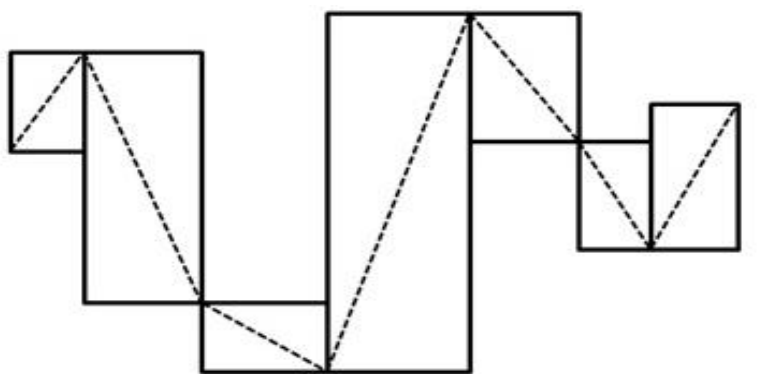

Figure 4 Showing that there will be $n-1$ disjoint rectangles 
Theorem 2.4. Let $\mathrm{P}$ be a set of $\mathrm{n}$ points in general position. There will be at least $n-1$ disjoint axis-parallel rectangles in S.

Proof. We assume that no two points in $\mathrm{P}$ have the same $\mathrm{x}$ or $\mathrm{y}$ coordinates. Sort the points of $\mathrm{P}$ with respect to $\mathrm{x}$ coordinate. Then the rectangles corresponding to consecutive pairs (refer Figure 4) in sorted list shall be disjoint. So there will be at least $\mathrm{n}-1$ disjoint rectangles.

Theorem 2.5. For a point set $P$. where no two points have the same $\mathrm{x}$ or y coordinate. We derive a 2 -factor approximation algorithm for the size of stabbing set of $\left(\begin{array}{l}n \\ 2\end{array}\right)$ Axis-parallel induced by each pair of point's a.b $\in \mathrm{P}$ as the diagonal of the rectangles.

Proof. Replace Theorem 2.3 with Theorem 2.5 in the proof of Theorem 2.4 to get the result. This problem ends with the following comment. Finding The minimum stabbing set of arbitrary rectangles in NP-Complete. We do not have any hardness result for the special case of rectangles considered here; neither do we have any polynomial time algorithm. Nielsen[9] presented an $\mathrm{O}(\log \mathrm{n})$-approximation algorithm for finding the stabbing set of axis-parallel rectangles.

\section{CONCLUSION}

Stabbing through rectangle is done on that point when the stores affect the market in straight line method or in rectangle method. So this can help in getting the result in better way as for the brand store 2 on that area where the store 1 is already established there market, as these are the points or marks that can effect. From the theorem 4.2 it is clear that $2 n-2$ stabbing points are always sufficient to stab all the rectangles of $S$ for the point set $\mathrm{P}$ having $\mathrm{n}$ points in general position. As for comparison we take the Aronov et al. results and our results that come out. We can compare this problem by taking different values $\mathrm{n}$ in the given theorem that can help in getting the result better to other ones. Here we take the value of $n$ equal to 4 for comparison the result. As our value $2 n-2-k$ is in which $\mathrm{n}$ is the number of points and $\mathrm{k}$ is the convex points. Convex points are those points which are present on the boundary of the graphs in which all the point comes inside that points.

Let there are 4 points to calculate the minimum number of stabbing point as shown in the figure 5(a). As the points are in the same plane then according to the Aronav et al. minimum number of stabbing points is $\mathrm{n}-1$.

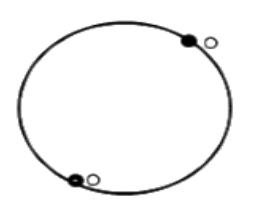

Figure 5(a) Four points for stabbing

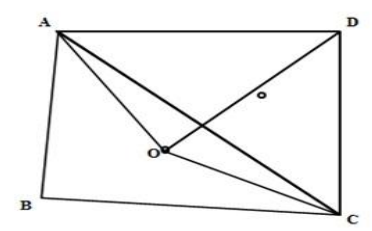

Figure 5(b) Four points for stabbing
As the $\mathrm{n}=4$ so, the stabbing points according to Aronov is 3.If the stabbing is done using for the 4 points in the same plane in general position using disks defined by pairs of points as diameter as define in the proposed work than the minimum number of stabbing points are $2 \mathrm{n}-2-\mathrm{k}$. Where $\mathrm{k}$ is the convex points For the problem $\mathrm{k}$ is 4 and $\mathrm{n}$ is also 4 or the minimum number of stabbing points are the 2 it is less than number of stabbing points calculating using aronov's method or the proposed method is better than Aronov et. al. method. As there we compare both the result by taking the $n$ equal to 4 and after that we get the better one. So we can say that most of the time our result is better than the other ones. This can help the brand store 2 to open the shop in the area where the brand store 1 is already established there market. So these are the stabbing point where the brand store 2 affects much more the market of brand store 1.Another example is for taking $n$ (points) equal to 5 .According to previous method the stabbing point is n-1 i.e. 5-1 equal to 4.So the stabbing point is 4 and that is near to the already existing point.

Now we can solve this problem with our algorithm putting $n$ equal to 5.By using the value $2 \mathrm{n}-2-\mathrm{k}$, $\mathrm{k}$ equal to 5 ,

So the stabbing point is 3 that is better than the previous one and it can cover more area of the other brand store.

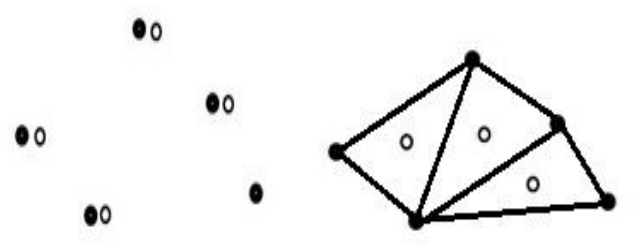

Figure 6 Five points stabbing

\section{RESULTS}

Now it is clear that when $\mathrm{n}$ shops of Store 1 are in the plane $\mathrm{P}$ having $\mathrm{k}$ shops at the convex hull position than the minimum number of shops is $2 \mathrm{n}-2-\mathrm{k}$ of Store 2 are the best to be open and when all the shops are at convex position than the best number of shops is $n-2$. So, Store 2 will covers the maximum area and easily interrupt the Store1.

We also proved the stabbing method by the help of rectangles induced by point as diagonal. The given method is also used for stabbing the brand store 2 to open there shops in the area of brand store 1 . The upper and lower bounds of the size of stabbing set are $2 n-2$ and $n-1$ respectively, for the axis parallel rectangles induced by the pair of points as diagonal. We have given an algorithm for stabbing sets for the axis parallel rectangles, when the points are in convex position. Convex position is the point which can cover all the points inside there line segment. We have also given an algorithm for stabbing sets for the axis parallel rectangles, when the points are in general position.

As the brand store 2 can stab there shop as according to different way of stabbing methods we discussed here for stabbing the brand store 1 in that area by treating the brand store 1 as the point and taking there value as $\mathrm{n}$. So by putting the valve of $\mathrm{n}$ in formulas we can easily get the result. As far as it concerned that this formula varies as according to the position of points that vary because there are lot of assumption that should be taken to implement this formula. 


\section{FUTURE SCOPE}

Further work which can be done to improve the bounds given in this thesis and the development of algorithms to find optimal size of stabbing sets for disks and rectangles. As there are no general algorithm for all the points there is different algorithm as according to the position of the points it can vary time to time. If the points are not in general position than there is other formula is to be calculated.

There are lots of assumptions in this so in future we can get the generalized algorithm to improve the stabbing point. In future the stabbing points values are to be minimized by which the better result come. So there is future scope of improving the upper bound or lower bound.

\section{REFERENCES}

[1] Boris Aronow, Muriel Dulieu, and ferran Hurtado, "Witness Gabriel graphs", 2010.

[2] Boris aronov, Muriel dulieu, and ferranhurtado, "Witness Delaunay graphs", Computational geometry: theory and application 44:329:344,2011.

[3] M.D. Berg, O.Cheong, M. Kreveld, and M. Overmars, "Computational geometry", Algorithms and Application Springer 3rd edition, 2008.
[4] G. Di Battista, p. edes, R. tamassia, and I.G. Tollis., "Graph drawing. Algorithms For the visualization of graphs", Prentice hall, 1999.

[5] Robert J. Fowler, Mike Paterson and steven L. Tanimoto, "Optimal packing and covering in the plane are npcomplete”,Inf process. Lett.,12(3):133-137, 1981.

[6] K. R. Gabriel and R.r.sokal, "A new statistical approach to geographic variation analysis", Systematic Zoology, 18:259-278, 1969.

[7] J. W. Jaromczyk and G.T. Toussaint, "Relative neighbourhood graphs and their relatives", Proc. IEEE, 80:1502-1517,1992.

[8] G. Liotta, "Proximity drawings", In inrr. Tamassia, editor, handbook of graphs drawing and visualization . CRC press.

[9] Frank Nielsen,"Fast stabbing of boxes in high dimension", Theoretical computer science, 246(1/2):53$75,2000$.

[10] G. T. Toussaint, "Geometric proximity graphs for improving nearest neighbor methods in instance based learning and data mining", International Journal of Computer Geom. And Applications. 15(2).

[11] G. T. Toussaint, "Some unsolved problems on proximity graphs", 1991 